\title{
Novel 2-(w-phosphonooxy-2-oxaalkyl)acrylate monomers for self-etching self-priming one part adhesive
}

\author{
Joachim E. Klee* and Uwe Lehmann
}

\author{
Full Research Paper \\ Address: \\ Dentsply De Trey, De-Trey-Str. 1, 78467 Konstanz, Germany \\ Email: \\ Joachim E. Klee* - joachim.klee@dentsply.com \\ * Corresponding author \\ Keywords: \\ adhesion to enamel and dentin; hydrolysis stable \\ 2-( $\omega$-phosphonooxy-2-oxaalkyl)acrylates; phosphorylation using \\ $\mathrm{POCl}_{3}$; polymerization enthalpy; shear bond strength
}

\author{
Beilstein J. Org. Chem. 2010, 6, 766-772. \\ doi:10.3762/bjoc.6.95 \\ Received: 04 May 2010 \\ Accepted: 09 August 2010 \\ Published: 07 September 2010 \\ Guest Editor: H. Ritter \\ (C) 2010 Klee and Lehmann; licensee Beilstein-Institut. \\ License and terms: see end of document.
}

\begin{abstract}
Novel hydrolysis stable 2-( $\omega$-phosphonooxy-2-oxaalkyl)acrylate monomers 3 with phosphoric acid moieties were synthesized by a three step synthesis via Baylis-Hillman reaction of ethyl acrylate and formaldehyde, and subsequent etherification of the obtained product with diols and phosphorylation using $\mathrm{POCl}_{3}$. The polymerization enthalpy of 2-( $\omega$-phosphonooxy-2-oxaalkyl)acrylates 3 as measured by DSC ranges from -29 to $-53 \mathrm{~kJ} \cdot \mathrm{mol}^{-1}$. The shear bond strength of adhesive compositions 4 , comprising of polymerizable acids 3, ranges from 5.8 to 19.3 $\mathrm{MPa}$ on enamel and from 8.7 to $16.9 \mathrm{MPa}$ on dentin.
\end{abstract}

\section{Introduction}

Dental adhesives have been employed for fixation of direct and indirect restorations since the 80 s of the last century. The first of these adhesives were composed of three-part systems, consisting of an etch gel, a primer and a bonding. Each of these adhesive parts was applied step-by-step in a relatively time consuming procedure that is prone to failure during the procedure. Therefore, a reduction of the complexity of the adhesives during application was desirable. Furthermore, the adhesion procedure should be more safe, easy and robust. In order to fulfill these demands some generations of adhesives were developed which combined the etch and prime function or the prime and bond function together in one part.
The first self-etching, self-priming dental adhesives were composed of two-part systems for stability reasons, i.e., low hydrolysis stability of conventional polymerizable acidic ester monomers and adhesive monomers in water or water/solvent mixtures. Newer one-part systems are based on conventional polymerizable acidic ester monomers and adhesive monomers, and these must be stored in a refrigerator to guarantee stability.

Today the demand is to have a one-part self-etching adhesive, which can be stored under ambient conditions and which combines all three steps of the adhesive procedure in one. Besides polymerizable monomers and acidic monomers, water 


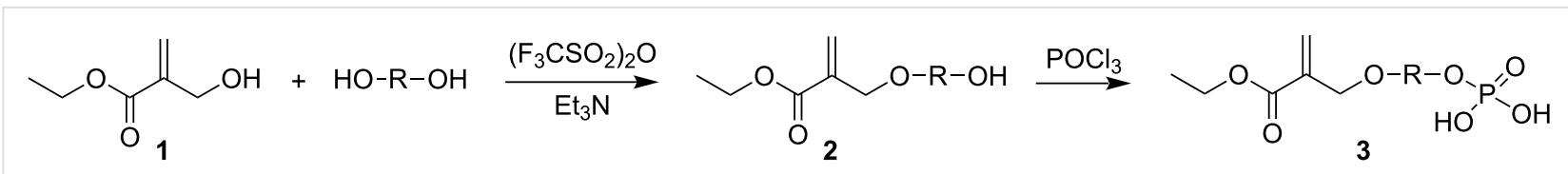

Scheme 1: Synthesis of novel ethyl 2-( $\omega$-phosphonooxy-2-oxaalkyl)acrylates 3.

is required in such a system for the acidic etch function. Therefore, such adhesives require novel hydrolysis stable monomers and novel hydrolysis stable acidic monomers.

Recently, some interesting approaches to acidic monomers were developed based on derivatives of $\alpha$-hydroxymethylacrylate esters [1], with one [2-4] or two phosphonic acid moieties [5], or with phosphoric [4,6] or sulfonic acid groups [7] as well as with carboxylic acid groups [7]. These acidic monomers are constructed in such a way that no hydrolysis sensitive moiety is present between the polymerizable moiety and the acidic group. The latter etches enamel and dentin surface, and is an anchor group, especially on dentin, due to the formation of calcium phosphate linkages.

The aim of the present investigation is the synthesis of novel polymerizable phosphoric acid ester monomers and their comparison concerning their adhesive performance in dental formulations. Furthermore, the aim is to clarify whether, under strong acidic conditions, hydrolysis of phosphoric acid ester moieties takes place in a similar manner to carboxylic acid esters, and to establish whether acidic molecules with 2-( $\omega$-phosphonooxy-2oxaalkyl)acrylate moieties exhibit the same adhesive performance as their ester analogues.

\section{Results and Discussion}

A series of novel 2-( $\omega$-phosphonooxy-2-oxaalkyl)acrylates 3, comprising of phosphoric acid ester moieties, were synthesized via a three step synthesis via Baylis-Hillman reaction of ethyl acrylate and formaldehyde, and subsequent etherification of the obtained product with diols and phosphorylation using $\mathrm{POCl}_{3}$ (Scheme 1, Table 1).

The double bonds in 3 are evident in the IR spectrum at 1637 (3a) and $1639 \mathrm{~cm}^{-1}(\mathbf{3 e}, \mathbf{3 h}, \mathbf{3 i})$ and in the ${ }^{1} \mathrm{H}$ NMR spectrum at $5.87 / 6.27 \mathrm{ppm}$ (3a). In the ${ }^{13} \mathrm{C}$ NMR spectra signals of the $\mathrm{sp}^{2}$ hybridized $\mathrm{C}$-atoms of the double bonds appear at 127.11/ $136.281 \mathrm{ppm}$ (3a). Signals of the $\mathrm{P}-\mathrm{OH}$ moiety were found in the ${ }^{1} \mathrm{H}$ NMR spectrum at $10.71 \mathrm{ppm}$ (3a) and in the ${ }^{31} \mathrm{P}$ NMR spectra at $0.16 \mathrm{ppm}(\mathbf{3 h})$ and $-0.11 / 0.07 \mathrm{ppm}(\mathbf{3 i})$.

\section{DSC Investigation of 3}

The polymerization behavior of 2-( $\omega$-phosphonooxy-2oxaalkyl)acrylates 3 was investigated by photo-polymerization using a DSC 7/DPA 7 unit. The polymerization enthalpy of 2-( $\omega$-phosphonooxy-2-oxaalkyl)acrylates 3 (Table 2 ) ranged from -29 to $-53 \mathrm{~kJ} \cdot \mathrm{mol}^{-1}$ per double bond, which is rather comparable to the polymerization enthalpy of methacrylic esters $\left(-52.8\right.$ to $\left.-59.9 \mathrm{~kJ} \cdot \mathrm{mol}^{-1}\right)[8-10]$ and lower compared to the

Table 1: Yields, viscosities and refraction indexes of molecules $\mathbf{2} \mathbf{a}-\mathbf{2} \mathbf{i}$ and $\mathbf{3 a} \mathbf{a} \mathbf{3} \mathbf{i}$.<smiles>[R]OOCC(=C)C(=O)OCC</smiles>

2<smiles>[H][R](=O)(O)OCC(=C)C(=O)OCC</smiles>

3

\begin{tabular}{cccccccc}
\hline & $\mathrm{R}$ & & $\begin{array}{c}M_{\mathrm{n}} \\
\mathrm{g} / \mathrm{mol}\end{array}$ & $\begin{array}{c}\text { Yield 2 } \\
\mathrm{g}(\%)\end{array}$ & $\begin{array}{c}\text { Yield 3 } \\
\mathrm{g}(\%)\end{array}$ & $\begin{array}{c}\eta \\
\mathrm{Pa} \text { s }\end{array}$ & $n_{D}^{20}$ \\
\hline $\mathrm{a}$ & $\left(\mathrm{CH}_{2}\right)_{2}$ & $\mathrm{C}_{8} \mathrm{H}_{15} \mathrm{O}_{7} \mathrm{P}$ & 254.18 & $10.12(50.0)$ & $14.2(55.0)$ & $40.13 \pm 0.62$ & $1.4760 \pm 0.002$ \\
b & $\left(\mathrm{CH}_{2}\right)_{3}$ & $\mathrm{C}_{9} \mathrm{H}_{17} \mathrm{O}_{7} \mathrm{P}$ & 268.20 & $37.28(21.4)$ & $31.0(58.8)$ & $4.01 \pm 0.10$ & $1.4662 \pm 0.001$ \\
$\mathrm{c}$ & $\left(\mathrm{CH}_{2}\right)_{6}$ & $\mathrm{C}_{12} \mathrm{H}_{23} \mathrm{O}_{7} \mathrm{P}$ & 310.28 & $16.01(36.2)$ & $8.8(44.5)$ & $2.04 \pm 0.05$ & $1.4652 \pm 0.0003$ \\
d & $\left(\mathrm{CH}_{2}\right)_{10}$ & $\mathrm{C}_{16} \mathrm{H}_{31} \mathrm{O}_{7} \mathrm{P}$ & 366.39 & $40.49(40.9)$ & $11.5(20.9)$ & - & - \\
e & $\left(\mathrm{CH}_{2}\right)_{10}{ }^{\mathrm{a}}$ & $\mathrm{C}_{14} \mathrm{H}_{27} \mathrm{O}_{7} \mathrm{P}$ & 338.34 & - & $8.6(60.0)$ & - & - \\
$\mathrm{f}$ & $\left(\mathrm{CH}_{2}\right)_{12}$ & $\mathrm{C}_{18} \mathrm{H}_{35} \mathrm{O}_{7} \mathrm{P}$ & 394.45 & $7.07(8.8)$ & $7.8(91.0)$ & - & - \\
$\mathrm{g}$ & $\mathrm{CH}_{2} \mathrm{CH}_{2} \mathrm{OCH}_{2} \mathrm{CH}_{2}$ & $\mathrm{C}_{10} \mathrm{H}_{19} \mathrm{O}_{8} \mathrm{P}$ & 298.23 & $19.45(46.0)$ & $17.0(65.0)$ & $6.26 \pm 0.16$ & $1.4691 \pm 0.002$ \\
$\mathrm{~h}$ & $\left(\mathrm{CH}_{2} \mathrm{CH}_{2} \mathrm{O}_{2} \mathrm{CH}_{2} \mathrm{CH}_{2}\right.$ & $\mathrm{C}_{12} \mathrm{H}_{23} \mathrm{O}_{9} \mathrm{P}$ & 342.28 & $28.34(46.9)$ & $31.1(88.6)$ & $4.16 \pm 0.05$ & $1.4692 \pm 0.0002$ \\
$\mathrm{i}$ & $\left(\mathrm{CH}_{2} \mathrm{CH}_{2} \mathrm{O}\right)_{3} \mathrm{CH}_{2} \mathrm{CH}_{2}$ & $\mathrm{C}_{14} \mathrm{H}_{27} \mathrm{O}_{10} \mathrm{P}$ & 386.34 & $51.91(73.5)$ & $32.9(50.2)$ & $2.35 \pm 0.01$ & $1.4702 \pm 0.0003$ \\
\hline
\end{tabular}

\footnotetext{
a 3 with free carboxylic acid moiety.
} 
polymerization enthalpy of acrylic esters $(-77.5$ to -80.5 $\mathrm{kJ} \cdot \mathrm{mol}^{-1}$ ) $[9,10]$. This may be due to the substitution of the double bond in $\mathbf{3}$, which is comparable with a methacrylic moiety rather than with an acrylic group.

\begin{tabular}{ccc} 
Table 2: Polymerization enthalpy $\Delta_{\mathrm{R}} \mathrm{H}$ of 3a-3i. \\
3 & $\mathrm{R}$ & $\begin{array}{c}\Delta_{\mathrm{R}} \mathrm{H} \\
\mathrm{kJ} / \mathrm{mol}\end{array}$ \\
\hline a & $\left(\mathrm{CH}_{2}\right)_{2}$ & -30.7 \\
b & $\left(\mathrm{CH}_{2}\right)_{3}$ & -45.7 \\
c & $\left(\mathrm{CH}_{2}\right)_{6}$ & -44.8 \\
d & $\left(\mathrm{CH}_{2}\right)_{10}$ & -45.1 \\
e & $\left(\mathrm{CH}_{2}\right)_{10}{ }^{a}$ & -53.2 \\
f & $\left(\mathrm{CH}_{2}\right)_{12}$ & -52.0 \\
g & $\mathrm{CH}_{2} \mathrm{CH}_{2} \mathrm{OCH}_{2} \mathrm{CH}_{2}$ & -29.3 \\
h & $\left(\mathrm{CH}_{2} \mathrm{CH}_{2} \mathrm{O}\right)_{2} \mathrm{CH}_{2} \mathrm{CH}_{2}$ & -41.8 \\
i & $\left(\mathrm{CH}_{2} \mathrm{CH}_{2} \mathrm{O}\right)_{3} \mathrm{CH}_{2} \mathrm{CH}_{2}$ & -50.8 \\
\hline
\end{tabular}

${ }^{a} \mathbf{3}_{\mathrm{e}}$ with free carboxylic acid moiety.

Obviously, molecules 3 with relatively short alkyl or oxyalkyl spacers exhibit the lowest polymerization enthalpy of -30.7 (3a) and $-29.3 \mathrm{~kJ} \cdot \mathrm{mol}^{-1}(\mathbf{3 g})$. The longer the spacers are, the higher is the polymerization enthalpy, up to -50.8 (3i) and $-52.0 \mathrm{~kJ} \cdot \mathrm{mol}^{-1}(\mathbf{3 f})$, which is only slightly lower than those of methacrylates. Probably, the steric proximity of the phosphoric acid moiety has an influence on the polymerization behavior due to the competitive reactions of protonation and radical formation of the amine co-initiator.

Assuming that there was complete polymerization at $80^{\circ} \mathrm{C}$, the polymerization enthalpy of $\mathbf{3 b}$ was measured under these conditions. It was found that the polymerization enthalpy increases from -45.7 at $37{ }^{\circ} \mathrm{C}$ to $-52.3 \mathrm{~kJ} / \mathrm{mol}$ at $80{ }^{\circ} \mathrm{C}$, which corresponds to a degree of double bond conversion of $87.3 \%$ at $37{ }^{\circ} \mathrm{C}$.

\section{Adhesion of phosphoric acids 3}

The shear bond strength of adhesive compositions 4 [11], comprising of polymerizable acids $\mathbf{3}$, ranges from 5.8 to 19.3 $\mathrm{MPa}$ on enamel and from 8.7 to $16.9 \mathrm{MPa}$ on dentin (Table 3, Figure 1). With increasing length of the aliphatic spacers of 3a-3f between the phosphoric acid ester and the ether linkage, adhesion both on enamel and dentin reach a maximum at $\mathbf{3 e}(n$ $=10 \mathrm{CH}_{2}$ moieties). Obviously, a balance of the hydrophobic and hydrophilic nature is achieved with $\mathbf{3 e}$, which is essential for adhesion on the very different substrates such as enamel and dentin.

Whilst the inclusion of larger oxymethylene moieties in $\mathbf{3}$ has no influence on the adhesion on dentin $(\mathbf{3 a}, \mathbf{3} \mathbf{g}-\mathbf{3 h})$, it has a pronounced effect on the adhesion on enamel which drops significantly with larger numbers of oxymethylene moieties.

Table 3: Adhesion of an adhesive composition 4, comprising of polymerizable acids 3 , on enamel and dentin.

\begin{tabular}{ccc}
\multicolumn{3}{c}{ Adhesion on } \\
$\mathbf{4}$ & enamel/MPa & dentin/MPa \\
\hline $\mathrm{a}$ & $10.9 \pm 3.0$ & $13.4 \pm 2.0$ \\
$\mathrm{c}$ & $10.8 \pm 1.4$ & $16.9 \pm 1.4$ \\
$\mathrm{~d}$ & $19.3 \pm 1.8$ & $15.1 \pm 2.2$ \\
$\mathrm{e}$ & $10.5 \pm 2.4$ & $23.7 \pm 3.4$ \\
$\mathrm{f}$ & $17.5 \pm 1.5$ & $8.7 \pm 1.0$ \\
$\mathrm{~g}$ & $12.6 \pm 2.2$ & $14.3 \pm 1.4$ \\
$\mathrm{~h}$ & $5.4 \pm 0.9$ & $13.3 \pm 3.4$ \\
$\mathrm{i}$ & $6.8 \pm 2.4$ & $13.4 \pm 1.3$ \\
\end{tabular}

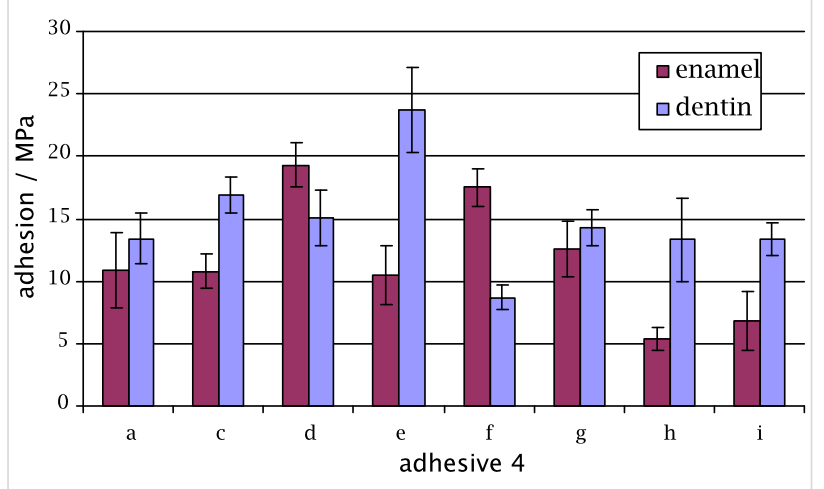

Figure 1: Adhesion of an adhesive composition 4, comprising of polymerizable acids 3 , on enamel and dentin.

\section{Conclusion}

Novel hydrolysis stable 2-( $\omega$-phosphonooxy-2-oxaalkyl)acrylate monomers 3 with phosphoric acid moieties and alkyl as well as oxyalkyl spacers were synthesized in three steps via Baylis-Hillman reaction of ethyl acrylate and formaldehyde, and subsequent etherification of the obtained product with diols and phosphorylation using $\mathrm{POCl}_{3}$. The polymerization enthalpy of 2-( $\omega$-phosphonooxy-2-oxaalkyl)acrylates 3 measured by DSC ranges from -29 to $-53 \mathrm{~kJ} \cdot \mathrm{mol}^{-1}$. The shear bond strength of adhesive compositions $\mathbf{4}$, comprising of polymerizable acids 3, ranges from 5.8 to $19.3 \mathrm{MPa}$ on enamel and from 8.7 to 16.9 $\mathrm{MPa}$ on dentin.

\section{Experimental}

The following commercial products were purchased from Sigma-Aldrich: formalin, ethyl acrylate, diazabicyclo[2.2.2]octane, dioxane, trifluoromethane sulphonic anhydride, dichloromethane, conc. $\mathrm{HCl}$, triethylamine, 1,2-ethanediol, 1,6- 
hexanediol, 1,8-octanediol 1,10-decanediol, 1,12-dodecanediol, diethylene glycol, triethylene glycol, tetraethylene glycol, phosphorous oxychloride, diethyl ether and 2,6-di-tert-butyl-4methylphenol Bis(2,4,6-trimethylbenzoyl)phenyl phosphine oxide was purchased from BASF Interorgana. $N, N^{\prime}$-bisacrylamido- $N, N^{\prime}$-diethyl-1,3-propane, and 3(4)8(9)-bis(acrylamidomethyl)tricyclo[5.2.1.0 $\left.0^{2,6}\right]$ decane were obtained from DENTSPLY. Spectrum TPH (DENTSPLY) is a dental composite comprising of methacrylate based resins and a glass filler.

\section{Ethyl 2-hydroxymethylacrylate (1)}

1 was prepared according to [1].

\section{Ethyl 2-(4-hydroxy-2-oxabutyl)acrylate (2a)}

To a solution of $32.52 \mathrm{~g}$ trifluoromethane sulphonic anhydride in $100 \mathrm{~mL}$ dichloromethane, a solution of $15 \mathrm{~g}(0.115 \mathrm{~mol}) \mathbf{1}$ and $11.66 \mathrm{~g}(0.115 \mathrm{~mol})$ triethylamine in $200 \mathrm{~mL}$ dichloromethane was added slowly at such a rate that the temperature of the reaction mixture remained below $5{ }^{\circ} \mathrm{C}$. The solution was then added dropwise at room temperature to $210 \mathrm{~g}(3.387 \mathrm{~mol})$ 1,2-ethanediol. After stirring for $12 \mathrm{~h}$ at room temperature, the solution was washed successively with $1 \times 200 \mathrm{~mL}$ water, $2 \times$ $250 \mathrm{~mL}$ of an aqueous sodium carbonate solution ( $25 \mathrm{wt} \%)$ and $1 \times 200 \mathrm{~mL}$ water. The organic layer was dried over magnesium sulfate and filtered. After the evaporation of the solvent, the oily crude product was stabilized with $15 \mathrm{mg}$ 2,6-di-tertbutyl-4-methylphenol and purified by vacuum distillation $\left(63{ }^{\circ} \mathrm{C} / 0.032 \mathrm{mbar}\right.$ ) to give $10.12 \mathrm{~g}$ (yield: $50 \%$ ) of $\mathbf{2 a}$ as a clear, colorless oil.

IR (film, cm $\left.{ }^{-1}\right) 3436(\mathrm{OH}), 2979,2931,2871\left(\mathrm{CH}_{3} / \mathrm{CH}_{2}\right), 1710$ (CO), $1638(\mathrm{C}=\mathrm{C}), 1453,1373,1304\left(\mathrm{CH}_{3} / \mathrm{CH}_{2}\right), 1270,1173$, 1109, 1052, 953. ${ }^{1} \mathrm{H}$ NMR (250 MHz, $\left.\mathrm{CDCl}_{3}, \mathrm{ppm}\right) 1.27$ (t, 3H, $\mathrm{CH}_{3}$ ), 2.52 (broad s, $\left.1 \mathrm{H}, \mathrm{OH}\right), 3.54-3.63\left(\mathrm{~m}, 2 \mathrm{H}, \mathrm{OCH}_{2} \mathrm{CH}_{2} \mathrm{O}\right)$, 3.67-3.78 (m, 2H, OCH $\left.\mathrm{CH}_{2} \mathrm{O}\right), 4.15-4.24\left(\mathrm{~m}, 4 \mathrm{H}, \mathrm{CH}_{2}\right.$ (1) and $\left.\mathrm{OCH}_{2} \mathrm{CH}_{3}\right), 5.84(\mathrm{~s}, 1 \mathrm{H}, \mathrm{CH}=\mathrm{C}), 6.28(\mathrm{~s}, 1 \mathrm{H}, \mathrm{CH}=\mathrm{C}) .{ }^{13} \mathrm{C}$ NMR (63 MHz, $\left.\mathrm{CDCl}_{3}, \mathrm{ppm}\right) 14.06\left(\mathrm{CH}_{3}\right), 60.74\left(\mathrm{OCH}_{2} \mathrm{CH}_{3}\right)$, $61.56\left(\mathrm{CH}_{2} \mathrm{OH}\right), 69.31\left(\mathrm{CH}_{2} \mathrm{CH}_{2} \mathrm{OH}\right), 71.80\left(=\mathrm{CCH}_{2} \mathrm{O}\right), 126.21$ $\left(\mathrm{CH}_{2}=\mathrm{C}\right), 137.03\left(\mathrm{C}=\mathrm{CH}_{2}\right), 165.82(\mathrm{CO})$.

\section{Ethyl 2-(4-phosphonooxy-2-oxabutyl)acrylate (3a)}

To a stirred solution of $15.46 \mathrm{~g}(0.1008 \mathrm{~mol})$ phosphorus oxychloride in $280 \mathrm{~mL}$ diethyl ether, a solution of $17.56 \mathrm{~g}$ $(0.1008 \mathrm{~mol}) \mathbf{2 a}$ and $10.2 \mathrm{~g}(0.1008 \mathrm{~mol})$ triethylamine in 250 $\mathrm{mL}$ diethyl ether was added dropwise at such a rate that the temperature of the reaction mixture remained below $5{ }^{\circ} \mathrm{C}$. After stirring for $14 \mathrm{~h}$ at room temperature, the mixture was filtered and then added slowly at $0{ }^{\circ} \mathrm{C}$ to $200 \mathrm{~mL}$ of water. The emulsion was stirred for $40 \mathrm{~min}$. The layers were separated and the aqueous layer was washed with $2 \times 100 \mathrm{~mL}$ diethyl ether. The aqueous layer was reduced in volume to $100 \mathrm{~mL}$ and extracted with $4 \times 100 \mathrm{~mL}$ dichloromethane. The combined organic extracts were dried over magnesium sulfate, filtered and evaporated to yield $19 \mathrm{~g}$ of a yellow oil. The oil was dissolved in 400 $\mathrm{mL}$ of water and washed with $3 \times 200 \mathrm{~mL}$ of diethyl ether. Concentration of the aqueous phase at reduced pressure and drying under vacuum ( $10^{-3} \mathrm{mbar}$ ) afforded $14.18 \mathrm{~g}$ (yield: $\left.55 \%\right)$ of a clear colorless oil, which was stabilized with $6.11 \mathrm{mg} 2,6-$ di-tert-butyl-4-methylphenol.

IR (film, cm ${ }^{-1}$ ) 3500-2500 broad absorption (OH), $2912\left(\mathrm{CH}_{3} /\right.$ $\left.\mathrm{CH}_{2}\right), 1709(\mathrm{CO}), 1637(\mathrm{C}=\mathrm{C}), 1456,1374\left(\mathrm{CH}_{3} / \mathrm{CH}_{2}\right), 1261$, 1178, 1105, 1014, 949. ${ }^{1} \mathrm{H}$ NMR (250 MHz, $\left.\mathrm{CDCl}_{3}, \mathrm{ppm}\right) 1.25$ (t, $3 \mathrm{H}, \mathrm{CH}_{3}$ ), 3.70 (broad s, $\left.2 \mathrm{H}, \mathrm{CH}_{2}\right), 4.30-4.13\left(\mathrm{~m}, 6 \mathrm{H}, \mathrm{CH}_{2}\right.$ ), $5.87(\mathrm{~s}, 1 \mathrm{H}, \mathrm{CH}=\mathrm{C}), 6.27(\mathrm{~s}, 1 \mathrm{H}, \mathrm{CH}=\mathrm{C}), 10.71($ broad s, $2 \mathrm{H}$, $\left.\mathrm{PO}_{3} \mathrm{H}_{2}\right) .{ }^{13} \mathrm{C} \mathrm{NMR}\left(63 \mathrm{MHz}, \mathrm{CDCl}_{3}, \mathrm{ppm}\right) 13.94\left(\mathrm{CH}_{3}\right), 60.94$ $\left(\mathrm{OCH}_{2} \mathrm{CH}_{3}\right), 66.01\left(\mathrm{OCH}_{2} \mathrm{CH}_{2}\right), 69.25\left(\mathrm{OCH}_{2} \mathrm{CH}_{2}\right), 69.40$ $\left(=\mathrm{CCH}_{2} \mathrm{O}\right), 127.11\left(\mathrm{CH}_{2}=\mathrm{C}\right), 136.28\left(C=\mathrm{CH}_{2}\right), 166.00(\mathrm{CO})$. ${ }^{31} \mathrm{P}$ NMR $\left(\mathrm{CD}_{3} \mathrm{OD} ; \mathrm{ppm}\right):-0.21 / 0.01\left(\mathrm{~d}, \mathrm{PO}_{3} \mathrm{H}_{2}\right)$.

$\mathbf{2 b}-\mathbf{2} \mathbf{i}$ and $\mathbf{3 b}-\mathbf{3 i}$ were synthesized as described for $\mathbf{2 a}$ and $\mathbf{3 a}$.

\section{Ethyl 2-(5-hydroxy-2-oxapentyl)acrylate (2b)}

bp 100-120 ${ }^{\circ} \mathrm{C} / 0.093$ mbar. IR (film, $\mathrm{cm}^{-1}$ ): 3500-2500 broad absorption (OH), $2877\left(\mathrm{CH}_{3} / \mathrm{CH}_{2}\right), 1705(\mathrm{CO}), 1637(\mathrm{C}=\mathrm{C})$, 1469, 1381, 1177, 1101, 1002, 953, 819, 746. ${ }^{1} \mathrm{H}$ NMR $(250$ $\mathrm{MHz}, d_{6}$-DMSO, ppm): $\delta=1.18\left(\mathrm{t}, 3 \mathrm{H}, \mathrm{CH}_{3}\right), 1,72-1,87(\mathrm{~m}$, $\left.2 \mathrm{H}, \mathrm{CH}_{2}\right), 3.43-3,51\left(\mathrm{~m}, 2 \mathrm{H}, \mathrm{CH}_{2}\right), 3.81-3,94\left(\mathrm{~m}, 2 \mathrm{H}, \mathrm{CH}_{2}\right)$, 4.00-4.19 (m, 4H, $\left.\mathrm{CH}_{2}\right), 5.79(\mathrm{~s}, 1 \mathrm{H}, \mathrm{CH}=\mathrm{C}), 6.12(\mathrm{~s}, 1 \mathrm{H}$, $\mathrm{CH}=\mathrm{C}) .{ }^{13} \mathrm{C}$ NMR $\left(63 \mathrm{MHz}, d_{6}\right.$-DMSO, ppm): $\delta=14.35$ $\left(\mathrm{CH}_{3}\right), 30.58,30.65\left(\mathrm{CH}_{2} \mathrm{CH}_{2} \mathrm{CH}_{2}\right), 60.79\left(\mathrm{OCH}_{2} \mathrm{CH}_{3}\right), 63.15$, $63.18\left(\mathrm{OCH}_{2} \mathrm{CH}_{2}\right), 66.88\left(\mathrm{OCH}_{2} \mathrm{CH}_{2}\right), 68.76\left(=\mathrm{CCH}_{2} \mathrm{O}\right)$, $125.77\left(\mathrm{CH}_{2}=\mathrm{C}\right), 137.84\left(C=\mathrm{CH}_{2}\right), 165.61(\mathrm{CO})$.

\section{Ethyl 2-(8-hydroxy-2-oxaoctyl)acrylate (2c)}

The substance was dried in vacuum without distillation. IR (film, $\mathrm{cm}^{-1}$ ): $3383(\mathrm{OH}), 2935 / 2866\left(\mathrm{CH}_{3} / \mathrm{CH}_{2}\right), 1710(\mathrm{CO})$, $1638(\mathrm{C}=\mathrm{C}), 1452,1383,1303,1269,1158,1100,1055,949$, 816.

\section{Ethyl 2-(12-hydroxy-2-oxadodecyl)acrylate (2d)}

bp $129-140{ }^{\circ} \mathrm{C} / 0.039$ mbar. IR (film, $\mathrm{cm}^{-1}$ ): 3425 (OH), 2926/ $2855\left(\mathrm{CH}_{3} / \mathrm{CH}_{2}\right), 1714(\mathrm{CO}), 1638(\mathrm{C}=\mathrm{C}), 1459 / 1375 / 1303$ $\left(\mathrm{CH}_{3} / \mathrm{CH}_{2}\right), 1270 / 1172 / 1102 / 1031 / 949 .{ }^{1} \mathrm{H} \mathrm{NMR}(250 \mathrm{MHz}$, $\left.\mathrm{CDCl}_{3}, \mathrm{ppm}\right): \delta=1.08-1.24\left(\mathrm{~m}, 15 \mathrm{H}, \mathrm{CH}_{2}, \mathrm{CH}_{3}\right), 1.24-1.49(\mathrm{~m}$, $4 \mathrm{H}, \mathrm{CH}_{2}$ ), 3.17 (broad s, $\left.1 \mathrm{H}, \mathrm{OH}\right), 3.27\left(\mathrm{t}, 2 \mathrm{H}, \mathrm{OCH}_{2}\right) 3.37$ (t, $\left.2 \mathrm{H}, \mathrm{OCH}_{2}\right), 3.96\left(\mathrm{~s}, 2 \mathrm{H}, \mathrm{CH}_{2}(1)\right), 4.01\left(\mathrm{q}, 2 \mathrm{H}, \mathrm{OCH}_{2} \mathrm{CH}_{3}\right) 5.65$ $(\mathrm{s}, 1 \mathrm{H}, \mathrm{CH}=\mathrm{C}), 6.07(\mathrm{~s}, 1 \mathrm{H}, \mathrm{CH}=\mathrm{C}) .{ }^{13} \mathrm{C} \mathrm{NMR}(63 \mathrm{MHz}$, $\left.\mathrm{CDCl}_{3}, \mathrm{ppm}\right): \delta=13.64\left(\mathrm{CH}_{3}\right), 25.36,25.68,28.98,29.07$, $29.11,29.17$ and $32.24\left(\mathrm{CH}_{2}(8-15)\right), 60.10\left(\mathrm{CH}_{2} \mathrm{OH}\right), 68.31$ $\left(\mathrm{OCH}_{2} \mathrm{CH}_{2}\right), 70.52\left(=\mathrm{CCH}_{2} \mathrm{O}\right), 124.69\left(\mathrm{CH}_{2}=\mathrm{C}\right), 137.12$ $\left(C=\mathrm{CH}_{2}\right), 165.37(\mathrm{CO})$. 


\section{Ethyl 2-(14-hydroxy-2-oxatetradecyl)acrylate (2f)}

Purification was performed by recrystallization from $\mathrm{CH}_{2} \mathrm{Cl}_{2}$ followed by column chromatography on silica gel $(115 \mathrm{~g})$ with $\mathrm{CH}_{2} \mathrm{Cl}_{2}$ as eluent. IR (film, $\mathrm{cm}^{-1}$ ): $3412(\mathrm{OH}), 2924 / 2855$ $\left(\mathrm{CH}_{3} / \mathrm{CH}_{2}\right), 1715(\mathrm{CO}), 1638(\mathrm{C}=\mathrm{C}), 1460 / 1375 / 1303\left(\mathrm{CH}_{3} /\right.$ $\left.\mathrm{CH}_{2}\right), 1270 / 1173 / 1103 / 1054 / 1029 / 951$.

\section{Ethyl 2-(7-hydroxy-2,5-dioxaheptyl)acrylate (2g)} bp $94-102{ }^{\circ} \mathrm{C} / 0.026$ mbar. IR (film, $\mathrm{cm}^{-1}$ ): $3426(\mathrm{OH}), 2871$ $\left(\mathrm{CH}_{3} / \mathrm{CH}_{2}\right), 1712(\mathrm{CO}), 1639(\mathrm{C}=\mathrm{C}), 1456,1374,1303,1270$, 1174, 1099, 1028, 953, 887, 816.

\section{Ethyl 2-(10-hydroxy-2,5,8-trioxadecyl)acrylate (2h)} bp $138-141^{\circ} \mathrm{C} / 0.041 \mathrm{mbar}$. IR (film, $\mathrm{cm}^{-1}$ ): $3433(\mathrm{OH}), 2964 /$ 2929/2874 $\left(\mathrm{CH}_{3} / \mathrm{CH}_{2}\right), 1711(\mathrm{CO}), 1638(\mathrm{C}=\mathrm{C}), 1453 / 1385 /$ $1302\left(\mathrm{CH}_{3} / \mathrm{CH}_{2}\right), 1269 / 1151 / 1099 / 1056 / 984 / 882 .{ }^{13} \mathrm{C} \mathrm{NMR}(63$ $\left.\mathrm{MHz}, \mathrm{CDCl}_{3}, \mathrm{ppm}\right): \delta=13.0\left(\mathrm{CH}_{3}\right), 60.3\left(\mathrm{CH}_{2} \mathrm{O}\right), 68.0-71.5$ $\left(\mathrm{CH}_{2}\right), 124.3\left(\mathrm{CH}_{2}=\mathrm{C}\right), 136.1\left(\mathrm{C}=\mathrm{CH}_{2}\right), 164.5(\mathrm{CO})$.

\section{Ethyl 2-(13-hydroxy-2,5,8,11-tetraoxatridecyl)acry- late (2i)}

bp $147-153^{\circ} \mathrm{C} / 0.037$ mbar. IR (film, $\mathrm{cm}^{-1}$ ): $3370(\mathrm{OH}), 2870$ $\left(\mathrm{CH}_{3} / \mathrm{CH}_{2}\right), 1713(\mathrm{CO}), 1639(\mathrm{C}=\mathrm{C}), 1458 / 1375\left(\mathrm{CH}_{3} / \mathrm{CH}_{2}\right)$, $1266\left(\mathrm{CH}_{2} \mathrm{O}\right), 1198 / 1028 / 947 / 816$.

\section{Ethyl 2-(5-phosphonooxy-2-oxapentyl)acrylate (3b)}

The compound was obtained as a clear, colorless oil. IR (film, $\left.\mathrm{cm}^{-1}\right)$ : 2926/2855 $\left(\mathrm{CH}_{3} / \mathrm{CH}_{2}\right), 1715(\mathrm{CO}), 1639(\mathrm{C}=\mathrm{C}), 1461 /$ $1375\left(\mathrm{CH}_{3} / \mathrm{CH}_{2}\right), 1265 / 1169 / 1101 / 1023 / 951$.

\section{Ethyl 2-(8-phosphonooxy-2-oxaoctyl)acrylate (3c)}

The compound was obtained as a clear, slightly yellow oil. IR (film, $\mathrm{cm}^{-1}$ ): 2934/2872 $\left(\mathrm{CH}_{3} / \mathrm{CH}_{2}\right), 1714(\mathrm{CO}), 1640(\mathrm{C}=\mathrm{C})$, $1456 / 1376\left(\mathrm{CH}_{3} / \mathrm{CH}_{2}\right), 1269 / 1170 / 1098 / 1027 / 950 .{ }^{1} \mathrm{H} \mathrm{NMR}$ $\left(250 \mathrm{MHz}, \mathrm{CD}_{3} \mathrm{OD}, \mathrm{ppm}\right): \delta=1.20-1.36\left(\mathrm{t}, 3 \mathrm{H}, \mathrm{CH}_{3}\right)$, 1.43-1.44 (m, $\left.4 \mathrm{H}, \mathrm{CH}_{2}\right), 3.30\left(\mathrm{~s}, 2 \mathrm{H}, \mathrm{CH}_{2}\right), 3.51\left(\mathrm{t}, 2 \mathrm{H}, \mathrm{CH}_{2}\right)$, 3.97-3.98 (m, $\left.2 \mathrm{H}, \mathrm{CH}_{2}\right), 4.99\left(\mathrm{~m}, 6 \mathrm{H}, \mathrm{CH}_{2} \mathrm{OPO}_{3} \mathrm{H}_{2}\right.$, $\left.\mathrm{OCH}_{2} \mathrm{CH}_{3}\right), 5.86(\mathrm{~s}, 1 \mathrm{H}, \mathrm{CH}=\mathrm{C}), 6.32(\mathrm{~s}, 1 \mathrm{H}, \mathrm{CH}=\mathrm{C}) \cdot{ }^{13} \mathrm{C}$ NMR (63 MHz, $\left.\mathrm{CD}_{3} \mathrm{OD}, \mathrm{ppm}\right): \delta=13.6\left(\mathrm{CH}_{3}\right), 26.5 / 26.9 / 30.6$ $\left(\mathrm{CH}_{2}\right), 31.4\left(\mathrm{CH}_{2} \mathrm{CH}_{2} \mathrm{OP}\right), 31.5\left(\mathrm{OCH}_{2} \mathrm{CH}_{2}\right), 60.8\left(\mathrm{CH}_{2} \mathrm{O}\right)$, $68.0\left(\mathrm{CH}_{2} \mathrm{OP}\right), 70.1\left(\mathrm{OCH}_{2} \mathrm{CH}_{2}\right), 71.8\left(=\mathrm{CCH}_{2} \mathrm{O}\right), 126.4$ $\left(\mathrm{CH}_{2}=\mathrm{C}\right), 139.8\left(\mathrm{C}=\mathrm{CH}_{2}\right), 169.5(\mathrm{CO}) .{ }^{31} \mathrm{P} \mathrm{NMR}\left(\mathrm{CD}_{3} \mathrm{OD}\right.$; ppm): $-0.36 / 0.14\left(\mathrm{~d}, \mathrm{PO}_{3} \mathrm{H}_{2}\right)$.

\section{Ethyl 2-(12-phosphonooxy-2-oxadodecyl)acrylate} (3d)

The compound was obtained as a yellowish solid. IR (film, $\left.\mathrm{cm}^{-1}\right)$ : 2926/2855 $\left(\mathrm{CH}_{3} / \mathrm{CH}_{2}\right), 1715(\mathrm{CO}), 1639(\mathrm{C}=\mathrm{C}), 1461 /$ $1375\left(\mathrm{CH}_{3} / \mathrm{CH}_{2}\right), 1265 / 1169 / 1101 / 1023 / 951 .{ }^{1} \mathrm{H}$ NMR $(250$ $\left.\mathrm{MHz}, \mathrm{CDCl}_{3}, \mathrm{ppm}\right): \delta=1.15-1.36\left(\mathrm{~m}, 15 \mathrm{H}, \mathrm{CH}_{3}\right), 1.45-1.77$ $\left(\mathrm{m}, 4 \mathrm{H}, \mathrm{CH}_{2}\right), 3.41\left(\mathrm{t}, 2 \mathrm{H}, \mathrm{CH}_{2}(3)\right), 4.03-4.24(\mathrm{~m}, 4 \mathrm{H}$,
$\left.\mathrm{CH}_{2} \mathrm{OPO}_{3} \mathrm{H}_{2}, \mathrm{OCH}_{2} \mathrm{CH}_{3}\right), 4.11\left(\mathrm{~s}, 2 \mathrm{H}, \mathrm{CH}_{2}\right), 5.79(\mathrm{~s}, 1 \mathrm{H}$, $\mathrm{CH}=\mathrm{C}), 6.22(\mathrm{~s}, 1 \mathrm{H}, \mathrm{CH}=\mathrm{C}) \cdot{ }^{13} \mathrm{C}$ NMR $\left(63 \mathrm{MHz}, \mathrm{CDCl}_{3}\right.$, ppm): $\delta=13.98\left(\mathrm{CH}_{3}\right), 25.08,25.93,28.92,29.19,29.25,29.30$ and $29.45\left(\mathrm{CH}_{2}(8-15)\right), 60.42\left(\mathrm{CH}_{2} \mathrm{CH}_{3}, \mathrm{CH}_{2} \mathrm{OP}\right), 68.63$ $\left(\mathrm{OCH}_{2} \mathrm{CH}_{2}\right), 70.86\left(=\mathrm{CCH}_{2} \mathrm{O}\right), 125.08\left(\mathrm{CH}_{2}=\mathrm{C}\right), 137.38$ $\left(C=\mathrm{CH}_{2}\right), 165.69(\mathrm{CO})$.

\section{2-(12-phosphonooxy-2-oxadodecyl)acrylic acid (3e)}

To an ice cold solution $\left(0-5^{\circ} \mathrm{C}\right)$ of $3.470 \mathrm{~g}(10.14 \mathrm{mmol})$ of $\mathbf{3 d}$ dissolved in $20 \mathrm{~mL}$ water, an aqueous solution of $1.217 \mathrm{~g}$ (30.41 mmol) sodium hydroxide dissolved in $12.165 \mathrm{~mL}$ of water was added slowly. The solution was then stirred for $24 \mathrm{~h}$ at ambient temperature. The basic solution was acidified with $25 \mathrm{~mL}$ of a $1 \mathrm{~N} \mathrm{HCl}$, saturated with $\mathrm{NaCl}$ and extracted three times with 50 $\mathrm{mL}$ THF. The extracts were dried over $\mathrm{Na}_{2} \mathrm{SO}_{4}$ and the solvent was removed. Yield: $2.01 \mathrm{~g}(62.8 \%)$, of a yellowish solid, $\mathrm{mp}$ $63.2{ }^{\circ} \mathrm{C}$

IR (film, $\left.\mathrm{cm}^{-1}\right)$ : 3500-3100 (COOH, broad), 2924/2854 $\left(\mathrm{CH}_{3} /\right.$ $\left.\mathrm{CH}_{2}\right), 1697(\mathrm{CO}), 1633(\mathrm{C}=\mathrm{C}), 1458 / 1373\left(\mathrm{CH}_{3} / \mathrm{CH}_{2}\right), 1173 /$ 1010/827. ${ }^{1} \mathrm{H}$ NMR $\left(250 \mathrm{MHz}, \mathrm{CDCl}_{3}, \mathrm{ppm}\right): \delta=1.95-2.18$ $\left(\mathrm{m}, 16 \mathrm{H}, \mathrm{CH}_{2}\right), 3.90\left(\mathrm{t}, 2 \mathrm{H}, \mathrm{CH}_{2} \mathrm{OPO}_{3} \mathrm{H}_{2}\right), 4.37-4.43(\mathrm{~m}, 2 \mathrm{H}$, $\left.\mathrm{OCH}_{2} \mathrm{CH}_{2}\right), 4.6\left(\mathrm{~s}, 2 \mathrm{H},=\mathrm{CCH}_{2} \mathrm{O}\right), 5,75(\mathrm{~s}, 1 \mathrm{H}, \mathrm{CH}=\mathrm{C}), 6.30(\mathrm{~s}$, $1 \mathrm{H}, \mathrm{CH}=\mathrm{C}) .{ }^{13} \mathrm{C}$ NMR $\left(63 \mathrm{MHz}, \mathrm{CDCl}_{3}, \mathrm{ppm}: \delta=25.08-31.6\right.$ $\left(\mathrm{CH}_{2}\right), 67.3\left(\mathrm{CH}_{2} \mathrm{OP}\right), 69.5\left(\mathrm{OCH}_{2} \mathrm{CH} 2\right), 71.2\left(=\mathrm{CCH}_{2} \mathrm{O}\right)$, $125.8\left(\mathrm{CH}_{2}=\mathrm{C}\right), 137.6\left(\mathrm{CH}_{2}=\mathrm{C}\right), 168.7(\mathrm{CO})$.

\section{Ethyl 2-(14-phosphonooxy-2-oxatetradecan)acry- late (3f)}

The compound was obtained as a white wax-like solid. IR (film, $\left.\mathrm{cm}^{-1}\right): 2918 / 2854\left(\mathrm{CH}_{3} / \mathrm{CH}_{2}\right), 1712(\mathrm{CO}), 1637(\mathrm{C}=\mathrm{C}), 1470 /$ $1379\left(\mathrm{CH}_{3} / \mathrm{CH}_{2}\right), 1304 / 1238 / 1165 / 1116 / 1026 / 853 .{ }^{1} \mathrm{H} \mathrm{NMR}$ $\left(250 \mathrm{MHz}, \mathrm{CD}_{3} \mathrm{OD}, \mathrm{ppm}\right): \delta=1.28-1.41\left(\mathrm{~m}, 17 \mathrm{H}, \mathrm{CH}_{3}\right)$, 1.58-1.69 (m, 4H, $\left.\mathrm{CH}_{2}\right), 3.48-3.51\left(\mathrm{t}, 2 \mathrm{H}, \mathrm{CH}_{2}\right), 3.94-3.98(\mathrm{q}$, $\left.2 \mathrm{H}, \mathrm{OCH}_{2} \mathrm{CH}_{3}\right), 4.15\left(\mathrm{~s}, 2 \mathrm{H}, \mathrm{CH}_{2}\right), 5.20\left(\mathrm{~s}, 2 \mathrm{H}, \mathrm{CH}_{2}\right), 5.85(\mathrm{~s}$, $1 \mathrm{H}, \mathrm{CH}=\mathrm{C}), 6.26(\mathrm{~s}, 1 \mathrm{H}, \mathrm{CH}=\mathrm{C}) .{ }^{13} \mathrm{C} \mathrm{NMR}\left(63 \mathrm{MHz}, \mathrm{CD}_{3} \mathrm{OD}\right.$, ppm): $\delta=15.50\left(\mathrm{CH}_{3}\right), 26.69,27.31,30.37,30.61,30.75,31.49$ and 31.56, $\left(\mathrm{CH}_{2}\right), 62.52\left(\mathrm{CH}_{2} \mathrm{CH}_{3}\right), 67.81,67.69\left(\mathrm{CH}_{2} \mathrm{OP}\right)$, $70.21\left(\mathrm{OCH}_{2} \mathrm{CH}_{2}\right), 71.99\left(=\mathrm{CCH}_{2} \mathrm{O}\right), 126.28\left(\mathrm{CH}_{2}=\mathrm{C}\right), 139.51$ $\left(C=\mathrm{CH}_{2}\right), 169.51(\mathrm{CO}) .{ }^{31} \mathrm{P}$ NMR $\left(\mathrm{CD}_{3} \mathrm{OD} ; \mathrm{ppm}\right):-0.57 / 0.13$ (d, $\mathrm{PO}_{3} \mathrm{H}_{2}$ ).

\section{Ethyl 2-(7-phosphonooxy-2,5-dioxaheptyl)acrylate} (3g)

The compound was obtained as a clear, colorless oil. IR (film, $\left.\mathrm{cm}^{-1}\right)$ : 2926/2878 $\left(\mathrm{CH}_{3} / \mathrm{CH}_{2}\right), 1712(\mathrm{CO}), 1640(\mathrm{C}=\mathrm{C}), 1453 /$ $1379\left(\mathrm{CH}_{3} / \mathrm{CH}_{2}\right), 1240 / 1169 / 1105 / 818 .{ }^{1} \mathrm{H}$ NMR $(250 \mathrm{MHz}$, $\left.\mathrm{CD}_{3} \mathrm{OD}, \mathrm{ppm}\right): \delta=1.30-1.38\left(\mathrm{~m}, 3 \mathrm{H}, \mathrm{CH}_{2} \mathrm{CH}_{3}\right), 1.70-1.85(\mathrm{~m}$, $\left.2 \mathrm{H}, \mathrm{CH}_{2}\right), 3.31-3.46\left(\mathrm{t}, 2 \mathrm{H}, \mathrm{CH}_{2}\right), 3.61-3.73(\mathrm{~m}, 4 \mathrm{H}$, $\left.\mathrm{OCH}_{2} \mathrm{CH}_{3}\right), 4.07-4.25\left(\mathrm{~m}, 2 \mathrm{H}, \mathrm{CH}_{2}\right), 4.88\left(\mathrm{~s}, 2 \mathrm{H}, \mathrm{CH}_{2}\right), 5.92$ $(\mathrm{s}, 1 \mathrm{H}, \mathrm{CH}=\mathrm{C}), 6.27(\mathrm{~s}, 1 \mathrm{H}, \mathrm{CH}=\mathrm{C}) \cdot{ }^{13} \mathrm{C}$ NMR $(63 \mathrm{MHz}$, 
$\left.\mathrm{CD}_{3} \mathrm{OD}, \mathrm{ppm}\right): \delta=14.47\left(\mathrm{CH}_{3}\right), 61.88\left(\mathrm{CH}_{2} \mathrm{CH}_{3}\right), 66.89 / 66.73$ $\left(\mathrm{CH}_{2} \mathrm{OP}\right), 70.4\left(\mathrm{CH}_{2} \mathrm{CH}_{2} \mathrm{O}\right), 70.48\left(\mathrm{OCH}_{2} \mathrm{CH}_{2}\right), 71.42$ $\left(=\mathrm{CCH}_{2} \mathrm{O}\right), 71.49\left(\mathrm{OCH}_{2} \mathrm{CH}_{2}\right), 126.65\left(\mathrm{CH}_{2}=\mathrm{C}\right), 139.01$ $\left(C=\mathrm{CH}_{2}\right), 164.72(\mathrm{CO}) .{ }^{31} \mathrm{P}$ NMR $\left(\mathrm{CD}_{3} \mathrm{OD} ; \mathrm{ppm}\right):-0.01 / 0.79$ (d, $\mathrm{PO}_{3} \mathrm{H}_{2}$ ).

\section{Ethyl 2-(10-phosphonooxy-2,5,8-trioxadecyl)acry- late $(3 \mathrm{~h})$}

The compound was obtained as a clear, colorless oil. IR (film, $\left.\mathrm{cm}^{-1}\right): 2874\left(\mathrm{CH}_{3} / \mathrm{CH}_{2}\right), 1711(\mathrm{CO}), 1639(\mathrm{C}=\mathrm{C}), 1459 / 1372$ $\left(\mathrm{CH}_{3} / \mathrm{CH}_{2}\right), 1246 / 982 / 820 .{ }^{1} \mathrm{H}$ NMR $\left(250 \mathrm{MHz}, \mathrm{CD}_{3} \mathrm{OD}, \mathrm{ppm}\right)$ : $\delta=1.28-1.32\left(\mathrm{~m}, 3, \mathrm{CH}_{3}\right), 3.67 / 3.72\left(\mathrm{~m}, 10 \mathrm{H}, \mathrm{CH}_{2}\right), 4.09-4.10$ (q, 2H, $\left.\mathrm{OCH}_{2} \mathrm{CH}_{3}\right), 4.21-4.23\left(\mathrm{t}, 2 \mathrm{H}, \mathrm{CH}_{2}\right), 5.34\left(\mathrm{~s}, 2 \mathrm{H}, \mathrm{CH}_{2}\right)$, $5.91(\mathrm{~s}, 1 \mathrm{H}, \mathrm{CH}=\mathrm{C}), 6.30(\mathrm{~s}, 1 \mathrm{H}, \mathrm{CH}=\mathrm{C}) .{ }^{13} \mathrm{C} \mathrm{NMR}(63 \mathrm{MHz}$, $\left.\mathrm{CD}_{3} \mathrm{OD}, \mathrm{ppm}\right): \delta=13.57\left(\mathrm{CH}_{3}\right), 61.95\left(\mathrm{CH}_{2} \mathrm{CH}_{3}\right), 66.86 / 66.91$ $\left(\mathrm{CH}_{2} \mathrm{OP}\right), 70.4\left(\mathrm{CH}_{2} \mathrm{CH}_{2} \mathrm{O}\right), 70.5\left(\mathrm{OCH}_{2} \mathrm{CH}_{2}\right), 71.19$ $\left(=\mathrm{CCH}_{2} \mathrm{O}\right), 71.60\left(\mathrm{OCH}_{2} \mathrm{CH}_{2}\right), 126.74\left(\mathrm{CH}_{2}=\mathrm{C}\right), 139.21$ $\left(C=\mathrm{CH}_{2}\right), 165.6(\mathrm{CO}) .{ }^{31} \mathrm{P} \mathrm{NMR}\left(\mathrm{CD}_{3} \mathrm{OD} ; \mathrm{ppm}\right): 0.81 /$ $0.01 /-0.81\left(\mathrm{~m}, \mathrm{PO}_{3} \mathrm{H}_{2}\right)$.

\section{Ethyl 2-(13-phosphonooxy-2,5,8,11-tetraoxa- tridecyl)acrylate (3i)}

The compound was obtained as a clear, colorless oil. IR (film, $\left.\mathrm{cm}^{-1}\right): 2879\left(\mathrm{CH}_{3} / \mathrm{CH}_{2}\right), 1714(\mathrm{CO}), 1639(\mathrm{C}=\mathrm{C}), 1465 / 1398$ $\left(\mathrm{CH}_{3} / \mathrm{CH}_{2}\right), 1258 / 1150 / 1026 / 796 .{ }^{1} \mathrm{H} \mathrm{NMR}(250 \mathrm{MHz}$, $\left.\mathrm{CD}_{3} \mathrm{OD}, \mathrm{ppm}\right): \delta=1.21-1.28\left(\mathrm{~m}, 3, \mathrm{CH}_{3}\right), 3.59 / 3.63(\mathrm{~m}, 14 \mathrm{H}$, $\mathrm{CH}_{2}$ ), 4.06-4.14 (q, 2H, OCH $\left.\mathrm{CH}_{3}\right), 4.21-4.23$ (t, $2 \mathrm{H}, \mathrm{CH}_{2}$ ), $5.34\left(\mathrm{~s}, 2 \mathrm{H}, \mathrm{CH}_{2}\right), 5.92 / 5.94(\mathrm{~s}, 1 \mathrm{H}, \mathrm{CH}=\mathrm{C}), 6.24 / 6.28(\mathrm{~s}, 1 \mathrm{H}$, $\mathrm{CH}=\mathrm{C}), 8.71\left(\mathrm{~s}, 2 \mathrm{H}, \mathrm{PO}_{3} \mathrm{H}_{2}\right) .{ }^{13} \mathrm{C} \mathrm{NMR}\left(63 \mathrm{MHz}, \mathrm{CDCl}_{3}\right.$, ppm): $\delta=13.7\left(\mathrm{CH}_{3}\right), 60.5\left(\mathrm{CH}_{2} \mathrm{CH}_{3}\right), 65.5\left(\mathrm{CH}_{2} \mathrm{OP}\right), 68.8$ $\left(\mathrm{CH}_{2} \mathrm{CH}_{2} \mathrm{O}\right), 69.3\left(\mathrm{OCH}_{2} \mathrm{CH}_{2}\right), 69.7\left(=\mathrm{CCH}_{2} \mathrm{O}\right), 127.9$ $\left(\mathrm{CH}_{2}=\mathrm{C}\right), 136.4\left(\mathrm{C}=\mathrm{CH}_{2}\right), 168.5(\mathrm{CO}) .{ }^{31} \mathrm{P} \mathrm{NMR}\left(\mathrm{CD}_{3} \mathrm{OD}\right.$; ppm): $-0.11 / 0.07\left(\mathrm{~d}, \mathrm{PO}_{3} \mathrm{H}_{2}\right)$.

Adhesive composition 4a: $1.5261 \mathrm{~g} N, N^{\prime}$-bisacrylamido-1,3propane, $2.2150 \mathrm{~g} \mathrm{3,(4),8,(9)-bis(acrylamidomethyl)-}$ tricyclo[5.2.1.0 $\left.0^{2,6}\right]$ decane, $0.5551 \mathrm{~g}$ of 3a, $0.2776 \mathrm{~g}$ 2-acrylamido-2-methylpropanesulfonic acid, $0.0337 \mathrm{~g}$ camphorquinone, $0.0849 \mathrm{~g}$ bis(2,4,6-trimethylbenzoyl)phenyl phosphine oxide and $0.0392 \mathrm{~g} 4$-( $N, N$-dimethylamino)benzoic acid ethyl ester were dissolved in a solvent mixture of $3.5201 \mathrm{~g}$ ethanol and $1.7484 \mathrm{~g}$ water.

Adhesive compositions $\mathbf{4 c - i}$ were prepared from $\mathbf{3 c}-\mathbf{i}$ in the same manner as described above.

The following procedure was applied prior to adhesion measurements:

- First the teeth were abraded with 200 and 500 grit abrasive paper.
- Then the teeth were stored at $37^{\circ} \mathrm{C}$ in water.

- The teeth were treated with adhesive formulation for $20 \mathrm{~s}$ and the solvents evaporated by an air stream for $10 \mathrm{~s}$.

- Light curing of adhesive layer for $20 \mathrm{~s}$.

- A polymerized Spectrum TPH body applied on the adhesive was cured on the teeth 3 times for $20 \mathrm{~s}$.

- Finally, the prepared teeth were stored in water at $37^{\circ} \mathrm{C}$ for $2 \mathrm{~h}$ before the measurement.

IR spectra were measured with a FT-IR spectrometer (Nicolet 6700 FT-IR spectrometer, Thermo Scientific). The ${ }^{1} \mathrm{H}$ NMR, ${ }^{13} \mathrm{C}$ NMR and ${ }^{31} \mathrm{P}$ NMR spectra were obtained with a Bruker AC $250 \mathrm{MHz}$ spectrometer. Viscosities were measured with a Bohlin-Rheometer CS-50 at $23{ }^{\circ} \mathrm{C}$.

The melting points were taken with a DSC 7 (Perkin-Elmer). Polymerization enthalpies were measured in the isothermal mode at $37{ }^{\circ} \mathrm{C}$ with a photo-calorimeter DSC 7/DPA 7 (PerkinElmer). The light intensity in the visible portion of the spectrum was $108 \mathrm{~mW} \cdot \mathrm{cm}^{-2}$. Each DSC experiment included a short dark period (typically $6 \mathrm{~s}$ ) and a subsequent illumination period. After the first run, an additional run was made using the polymerized material under the same experimental conditions. The subtraction of these runs from one another removed the effect of different baselines for the dark and the illumination periods. In the monomers were dissolved $0.3 \mathrm{~mol} \%$ camphorquinone and $0.35 \mathrm{~mol} \% 4$-( $N, N$-dimethylamino)benzoic acid ethyl ester.

\section{References}

1. Mathias, L. J.; Warren, R. M. Polym. Prepr. 1989, 30, 235.

2. Moszner, N.; Zeuner, F.; Fischer, U. K.; Rheinberger, V. Macromol. Chem. Phys. 1999, 200, 1062.

3. Moszner, N.; Zeuner, F.; Rheinberger, V. Hydrolysestabile und polymerisierbare Acrylphsophonsäuren. DE 19746 708, Oct 16, 1997.

4. Avci, D.; Mathias, L. J. J. Polym. Sci., Part A: Polym. Chem. 2002, 40, 3221. doi:10.1002/pola.10413

5. Erdmann, C.; Ziegler, S.; Nefgen, S.; Bolln, C.; Mühlbauer, W.; Lück, R. Dental material containing phosphonic acids. WO 02/02057 A1, July 3 , 2001.

6. Klee, J. E.; Lehmann, U.; Walz, U.; Liu, H. A polymerizable phosphoric acid ester derivative and a dental composition employing it. EP 1454 911, March 7, 2003.

7. Hoffmann, M.; Erdrich, A. Hydrolysestabile Monomere mit Säuregruppen. EP 1681283 A1, Dec 24, 2005.

8. Dainton, F. S.; Ivin, K. J.; Walmsley, D. A. G. Trans. Faraday Soc. 1960, 56, 1784. doi:10.1039/tf9605601784

9. Joshi, R. M. J. Polym. Sci. 1962, 56, 313. doi:10.1002/pol.1962.1205616404

10. Flammersheim, H.-J.; Klemm, E. Acta Polym. 1985, 36, 443. doi:10.1002/actp.1985.010360810

11. Klee, J. E.; Lehmann, U.; Walz, U. One-part self-etching, self-priming dental adhesive composition. EP 1548021 B1, Dec 23, 2003. 


\section{License and Terms}

This is an Open Access article under the terms of the Creative Commons Attribution License

(http://creativecommons.org/licenses/by/2.0), which permits unrestricted use, distribution, and reproduction in any medium, provided the original work is properly cited.

The license is subject to the Beilstein Journal of Organic Chemistry terms and conditions:

(http://www.beilstein-journals.org/bjoc)

The definitive version of this article is the electronic one which can be found at:

doi:10.3762/bjoc.6.95 